\title{
EL ITEM AB HISPALI CORDVBAM EN LA GEOGRAPHIA DE PTOLOMEO. UNA PROPUESTA DE INTERPRETACIÓN DEL MÉTODO CARTOGRÁFICO PTOLEMAICO
}

\author{
Javier Urueña Alonso \\ Universidad de Valladolid \\ uruenaalonso@gmail.com
THE ITEM AB HISPALI CORDVBAM IN THE PTOLEMY'S GEOGRAPHIA. A PROPOSAL TO INTERPRET THE PTOLEMAIC MAP-MAKING METHOD

\begin{abstract}
RESUMEN: En el presente estudio se analiza la ubicación que Ptolomeo dio a las poblaciones del Item ab Hispali Cordubam (vía VIII de Hispania del Itinerario de Antonino) en la Geographia. El objetivo de este análisis es revelar el sistema de localización empleado por el autor para su posicionamiento, sus posibles fuentes y sus métodos de cálculo. Para ello se realiza un examen crítico de las dos obras y se comparan las distancias deducidas de las coordenadas ptolemaicas con las transmitidas por el Itinerario de Antonino y la realidad.
\end{abstract}

PALABRAS CLAVE: Geographia de Ptolomeo, Itinerario de Antonino, Hispania, método cartográfico, Item ab Hispali Cordubam (vía VIII).

\begin{abstract}
This study analyzes the position given by Ptolemy to the towns of the Item ab Hispali Cordubam (VIII $^{\text {th }}$ itinerary of Hispania of the Antonine Itinerary) in his Geographia. The aim of this analysis is to reveal the locating system used by the author in order to place these towns, his sources and his calculation method. This study includes a critical examination of the texts and a comparison between the distances deduced from the Ptolemaic coordinates, those transmitted by the Antonine Itinerary, and the real ones.
\end{abstract}

KEYWORDS: Ptolemy's Geographia, Antonine Itinerary, Hispania, Map-making method, Item ab Hispali Cordubam (VIII ${ }^{\text {th }}$ itinerary)..

RECIBIDO: 12.06.2013. ACEPTADO: 26.04.2014 


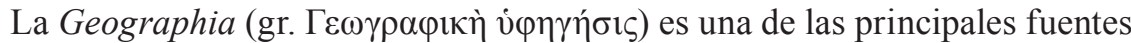
históricas para el análisis de la península Ibérica en la Antigüedad. El frío elenco de puntos ${ }^{1}$ y coordenadas que constituye la obra ha motivado un gran número de estudios; sin embargo, aún estamos lejos de comprender el método utilizado por Ptolomeo para su localización ${ }^{2}$.

El presente trabajo pretende contribuir a mejorar esta situación mediante el estudio comparativo de los datos recogidos en el Itinerario de Antonino, los expuestos por el autor alejandrino y la realidad, destacando las correspondencias y diferencias entre ellos. Dentro de esta línea analizamos la localización de los puntos documentados por el Itinerario de Antonino en la conocida como vía VIII de Hispania, Item ab Hispali Cordubam (Itin. Anton. Aug. 413.1-413.5).

Para la realización de este análisis seguimos los principios planteados por Müller (1883: 543) y Cuntz (1923: 110) para el estudio de la obra geográfica de Ptolomeo -recogidos por Gómez Fraile (2005) para Hispania-. Así, hemos calculado las distancias entre puntos a partir de las coordenadas dadas por Ptolomeo mediante la Ecuación $1^{3}$ y comparado los resultados con las medidas transmitidas

${ }^{1}$ En general, denominaremos como puntos a todos los elementos geográficos de longitud y latitud conocida, independientemente de su condición de población, puerto, cabo, río, monte, hito fronterizo....

${ }^{2}$ Entre los estudios sobre el sistema de localización de Ptolomeo se pueden señalar, entre otros, el propio de Marciano Heraclense, Periplus Maris Externi (vid. Müller 1855: 515-562), dentro de la historiografía clásica; el de Saavedra (2000), dentro de la moderna; los de Monteagudo (1947) y Martínez Hombre (1964), de los que parte la primera crítica sobre la metodología de este tipo de trabajos (Solana Sáinz 1972); y los más recientes de Montero Vítores (1991), Knapp (1993), Capalvo Liesa (1996), Canto (1997), Gómez Fraile (1997, 2005), Tornadijo Rodríguez (2008a, 2008b) y Tsorlini (2009).

${ }^{3}$ Los distintos autores que han calculado las distancias entre puntos en la obra de Ptolomeo han seguido diversas fórmulas. Así Tornadijo Rodríguez (2008a) sigue la misma fórmula que nosotros plantea-

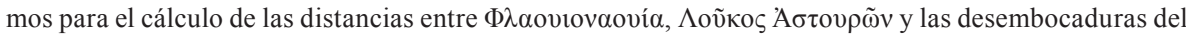

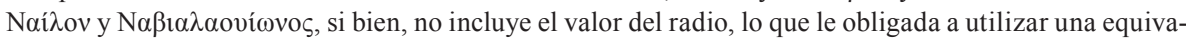
lencia imprecisa para la transformación de medidas angulares en lineales. El resto de autores consultados parte del teorema de Pitágoras para el cálculo de la distancia entre puntos (vid. Müller 1883; Blázquez 1892; Knapp 1993; Gómez Fraile 2005), sin embargo, la aplicación de este teorema sobre superficies esféricas es imprecisa y hasta cierto punto arbitraria, pues exige una determinación previa del grado latitudinal de referencia y el valor en él del grado de longitud, lo que puede condicionar en gran medida los resultados, si bien el error sólo es significativo en grandes distancias si se toma un valor de grado longitudinal medio entre las latitudes de los dos puntos:

$$
\left.\mathrm{d}\left(\mathrm{P}_{1}, \mathrm{P} 2\right)=\sqrt{ }\left(\left(\varphi_{1}-\varphi_{2}\right) \cdot \mathrm{g}_{\varphi}\right)^{2}+\left(\left(\lambda_{1}-\lambda_{2}\right) \cdot \mathrm{g}_{\lambda}\right)^{2}\right)
$$

Así, por ejemplo, Marciano Heraclense parte de la medida de 500 st para el grado de longitud, lo que distorsiona fuertemente sus resultados en latitudes alejadas del Ecuador. Capalvo declara partir de esta misma medida para la península Ibérica (1996: 90); sin embargo, de sus cálculos se deducen distintas medidas próximas a los 375 st. Knapp (1993: 105) parte de la medida de 400 st para el cálculo de las distancias entre las poblaciones de la Baetica, lo que sólo es válido en latitudes cercanas al paralelo $36^{\circ}$ -la medida exacta que considera Ptolomeo puede deducirse de los 72812 st que miden los $180^{\circ}$ del para-

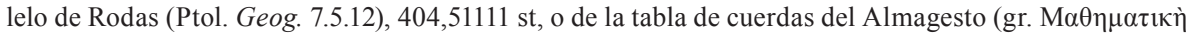
$\sigma v ́ v \tau \alpha \xi ı$ ) (Ptol. Alm. 1.11), donde puede calcularse una medida de 404,50926 st, distancia próxima a la real de 404,50672 st-. Gómez Fraile (2005: 39) parte de la medida de 375 st de la anotación del mapa de Hispania, que señala un valor del grado medio de longitud de la península Ibérica de 3/4 respecto al 
por el Itinerario de Antonino y las distancias en línea recta existentes entre los lugares donde se han reducido los distintos núcleos.

$$
\mathrm{d}\left(\mathrm{P}_{1}, \mathrm{P}_{2}\right)=\cos ^{-1}\left(\sin \varphi_{1} \cdot \sin \varphi_{2}+\cos \varphi_{1} \cdot \cos \varphi_{2} \cdot \cos \left(\lambda_{2}-\lambda_{1}\right)\right) \cdot \mathrm{r} \quad(\text { Ecuación } 1)^{4}
$$

El trabajo incluye un estudio crítico de los dos textos y considera las variantes de lectura de las medidas y de las coordenadas transmitidas evitando así las interpretaciones derivadas de otras ediciones ${ }^{5}$.

Este análisis nos permitirá comprender la ubicación de los puntos en la Geographia y profundizar en el sistema empleado por Ptolomeo para situar los distintos lugares.

\section{FUENTES Y MÉTODO DE LOCALIZACIÓN}

Ptolomeo declara valerse de dos tipos de fuentes para la localización de los puntos $^{6}$ : astronómicas, que incluyen la latitud y en muy pocos casos la longitud relativa ${ }^{7}$; y geométricas (corográficas e itinerarias), que señalan dimensiones, distancias entre puntos y posiciones relativas (Ptol. Geog. 1.2).

Las dos clases de fuentes tienen sus limitaciones. Los datos astronómicos, que generalmente son más precisos y transmiten la posición exacta de los puntos o permiten calcularla (Ptol. Geog. 1.2.5-1.3.5), son escasos y no siempre están bien determinados. Los geométricos, que son más numerosos y fiables, sólo transmiten distancias y posiciones relativas y, por ello, no permiten deducir una posición exacta de los puntos (Ptol. Geog. 1.2.4, 1.2.8).

grado de 500 st de los paralelos (Ptol. Geog. 8.4.1). Este valor corresponde aproximadamente al del grado de longitud en una latitud de $41^{\circ}, 377,3548$ st (Gómez Fraile 2005: 45) y, por tanto, es impreciso en latitudes septentrionales y meridionales de la Península.

${ }^{4} \mathbf{d}(\mathbf{P 1}$, P2): distancia entre el primer punto, P1 $(\varphi 1, \lambda 1)$, y el segundo, P2 $(\varphi 2, \lambda 2) \| \boldsymbol{\varphi 1}$ : latitud del primer punto || $\boldsymbol{\lambda 1}$ : longitud del primer punto $\| \boldsymbol{\varphi} 2$ : latitud del segundo punto $\| \boldsymbol{\lambda 2}$ : longitud del segundo punto $\| \mathbf{r}$ : radio ptolemaico de la Tierra $=(180000 \mathrm{st}) / 2 \pi \approx 282743,34$ st. Ptolomeo considera que la circunferencia de la Tierra mide 180000 st. Así se deduce del valor de 500 st de los grados de longitud en el Ecuador o de los grados de latitud $\left(360^{\circ} \cdot 500 \mathrm{st}\right.$ ) (Ptol. Geog. 1.7.1; 1.11.2), de los 90000 st que miden los $180^{\circ}$ de la tierra conocida en el Ecuador (Ptol. Geog. 7.5.12) o de las múltiples referencias a los valores de los grados de longitud en las distintas latitudes.

${ }^{5}$ La edición de Müller (1883) incluye diversas lecturas surgidas a partir de las interpretaciones del autor sobre las distancias entre puntos, lo que, en muchos casos, constituye una adaptación "forzada" del texto a la teoría.

${ }^{6}$ Estas informaciones procederían de la compilación realizada por Marino de Tiro (Ptol. Geog. 1.6), que Ptolomeo completa y rectifica (Ptol. Geog. 1.7-1.18) con las descripciones de "viajes" más recientes y fiables (Ptol. Geog. 1.5.2, 1.19.1), labor que debió ser considerable y que dio a la obra un carácter totalmente original (Knapp 1993: 104-105).

${ }^{7}$ El cálculo de estos parametros aparece recogido ampliamente en el Almagesto (Ptol. Alm. 2.2-2.5) y de forma breve en la Geographia (Ptol. Geog. 1.3.3, 1.4.2). 
El autor lograría superar estos condicionantes con la combinación de los dos tipos de fuentes. Así, completó el limitado número de puntos con informaciones astronómicas asociadas con aquellos de los que sólo disponía de datos geométricos y resolvió la imposibilidad de localizar de forma absoluta estos últimos partiendo de las posiciones de los anteriores (Ptol. Geog. 1.4.1-1.4.2) (Bunbury 1879: 554-555).

Para ello, Ptolomeo hubo de ubicar los puntos en dos fases. En un primer momento, situaría aquellos lugares con informaciones astronómicas asociadas constituyendo con ellos una red de referencia. En segundo término, ubicaría el resto de puntos a partir de las distancias itinerarias respecto a los puntos principales teniendo presentes las informaciones corográficas, etnográficas y administrativas, que los relacionaban directa o indirectamente.

La elaboración de la red de puntos de referencia partiría de un trabajo previo que hizo posible incluir en ella además de los lugares con latitud y longitud conocida, que serían escasos y en su mayoría estarían ubicados en el litoral ${ }^{8}$, aquellos de los que sólo conocía la latitud, más numerosos que los anteriores gracias a la facilidad de su cálculo y a las múltiples compilaciones existentes (Ptol. Geog. 1.4.2) (Gómez Fraile 2005: 38). El cálculo de la longitud de estos puntos hubo de realizarse aplicando el teorema de Menelao a superficies esféricas (Ptol. Alm. 1.13) (vid. Sidoli 2004: 126), cálculo que pudo realizar gracias a una tabla de cuerdas similar a la expuesta en el Almagesto (Ptol. Alm. 1.11).

Ptolomeo situaría todos estos lugares con coordenadas establecidas en una gratícula regional de paralelos y meridiano ${ }^{10}$ que, al menos para la península Ibérica, tuvo una precisión máxima de 5'. A partir de ellos esbozaría las grandes superficies geográficas: los continentes, las islas y las divisiones administrativas (Ptol. Geog. 2.1.6-2.1.11) (Knapp 1993: 106); lo que, posteriormente, le permitiría también determinar la posición relativa de algunas etnias y, con ello, la dirección general de las vías.

La localización de las poblaciones con informaciones geométricas, itinerarias y corográficas, posiblemente fuera la parte más intuitiva del trabajo del

\footnotetext{
${ }^{8}$ Dada la tradición de viajes marinos, la costa era la región más descrita y mejor conocida, como Ptolomeo destaca en el comentario de la obra de Marino de Tiro (Ptol. Geog. 1.18.6) (Knapp 1993: 105).

${ }^{9}$ La precisión de 30' de esta tabla puede explicar la posición de algunos puntos destacados del interior de la Península en coordenadas aparentemente redondeadas a medio segundo: Italica: $7^{\circ} \mathrm{E} 38^{\circ} \mathrm{N}$ (Ptol. Geog. 2.4.13); Scallabis: $6^{\circ} \mathrm{E} 41^{\circ} \mathrm{N}$ (Ptol. Geog. 2.5.7); Augusta Emerita: $8^{\circ} \mathrm{E} 39^{\circ} 30^{\prime} \mathrm{N}$ (Ptol. Geog. 2.5.8); Asturica Augusta: 9³0' E $44^{\circ} \mathrm{N}$ (Ptol. Geog. 2.6.36); Clunia: $11^{\circ} \mathrm{E} 42^{\circ} \mathrm{N}$ (Ptol. Geog. 2.6.56); Toletum: $10^{\circ} \mathrm{E} 41^{\circ} \mathrm{N}$ (Ptol. Geog. 2.6.57); Caesaraugusta: $14^{\circ} 30^{\prime} \mathrm{E} 41^{\circ} 30^{\prime} \mathrm{N}$ (Ptol. Geog. 2.6.63).

${ }^{10}$ Estas gratículas regionales pudieron estar realizadas con alguno de los sistemas de proyección descritos para el orbe (Ptol. Geog. 1.22-1.24) o sólo teniendo presente la proporción entre el paralelo central de cada mapa respecto a los meridianos (Ptol. Geog. 2.1.9-2.1.10), cuestión a la que el autor resta importancia para la elaboración de los mapas y, sin embargo, es fundamental para la deducción del valor de las distancias entre puntos. Así, si utilizó una de las primeras, previsiblemente la cónica simple, la distancia entre puntos puede calcularse aplicando la Ecuación 1 con un margen mínimo de error, y, si usó una gratícula rectilínea, el teorema de Pitágoras.
} 
autor; esto, sin embargo, no impidió la exactitud de la mayoría de las localizaciones $^{11}$. Para la ubicación de estos puntos utilizaría un sistema gráfico ${ }^{12}$, bien determinando su posición mediante la intersección de dos circunferencias con centro en sendos puntos de referencia de radio igual a las distancias respectivas al punto a ubicar ${ }^{13}$, bien mediante la intersección de una circunferencia con centro en un punto de referencia de radio igual a su distancia al punto a ubicar con la línea recta que esbozara el trazado general de la vía ${ }^{14}$, modificando, en ambos casos, el resultado al paralelo y meridiano de 5' más próximo que respetara la orientación del trayecto viario y las informaciones corográficas asociadas ${ }^{15}$.

\section{ESTUdio COMPARATIVO DEL ITINERARIO Y LA GEOGRAPHIA}

El Itinerario de Antonino (ITIN. Anton. Aug. 413-1-413.5) indica que la vía de Hispalis a Corduba tiene una distancia de 93 millas (aprox. 137,6 km) dividida en cuatro trayectos ${ }^{16}$ : entre Hispalis y Obucula, $42 \mathrm{mp}^{17}$; entre Obucula y Astigi, $15 \mathrm{mp}^{18}$; entre Astigi y Ad Aras, 12 mp; y, por último, entre Ad Aras y Corduba, 24 mp $^{19}$.

${ }^{11}$ Bunbury señala que la ubicación de estos puntos con coordenadas exactas es un intento de Ptolomeo de disfrazar su verdadera naturaleza imprecisa y las posibles correcciones arbitrarias que sobre ellos realiza siguiendo ideas preconcebidas (Bunbury 1879: 551). Sin embargo, como apunta Capalvo Liesa (1996: 90) y aquí constatamos, la mayoría de los puntos presumiblemente tomados de estas fuentes están bien ubicados en relación a los datos itinerarios que de ellas poseemos.

${ }^{12}$ La localización también pudo realizarse mediante operaciones aritméticas; sin embargo, la lentitud de su desarrollo unida a la ingente cantidad de puntos a ubicar nos hacen pensar que Ptolomeo se valió del relativamente rápido sistema gráfico.

${ }^{13}$ Este método explicaría por qué algunas de las distancias de las poblaciones intermedias respecto al primer punto de referencia, generalmente el origen de la vía, son inferiores a la documentada por el Itinerario y superiores respecto al segundo.

${ }^{14}$ Este sistema es más impreciso y conlleva la acumulación de los errores de redondeo, es posible, por tanto, que su uso se limitara a los trayectos de los que sólo se dispusiera de un punto de referencia.

${ }^{15}$ Ptolomeo condicionó las informaciones itinerarias a las étnicas (Capalvo Liesa 1996: 98-99) lo que explica porqué algunos puntos del mismo trayecto viario no se disponen en línea recta entre los lugares de referencia.

${ }^{16}$ El sector entre Hispalis y Astigi es el mismo que en la vía X, Item ab Hispali Emeritam (ItiN. Anton. Aug. 414-415), si bien ésta incorpora Carmo entre Hispalis y Obucula manteniendo las distancias, así: de Hispalis a Carmo, $22 \mathrm{mp}$; de Carmo a Obucula, $20 \mathrm{mp}$; y de Obucula a Astigi, $15 \mathrm{mp}$. El Anonymum Rauennate también incluye Carmo en la enumeración de las poblaciones de este sector (RAVEnN. 315.3-315.5): Astigin, Obucula, Carmone. Los Vasos de Vicarello (CIL XI 3281-3284) describen esta misma vía incluyendo Carmo y manteniendo las distancias, salvo para el trayecto de Ad Aras a Corduba, para el que el Itinerario da una distancia de $24 \mathrm{mp}$ y los Vasos de $23 \mathrm{mp}$, así: de Hispalis a Carmo, $22 \mathrm{mp}$; de Carmo a Obucla, $15 \mathrm{mp}$; de Obucla a Astigi, $15 \mathrm{mp}$; de Astigi a Ad Aras, $12 \mathrm{mp}$; de Ad Aras a Corduba, 23 mp; el Vaso IV no señala Ad Aras y da una distancia entre Astigi y Corduba de $35 \mathrm{mp}$. nota 16$)$.

${ }^{17}$ El trayecto debía incorporar la población de Carmo a $22 \mathrm{mp}$ de Hispalis y $20 \mathrm{mp}$ de Obucula (vid.

${ }^{18}$ La variante de $16 \mathrm{mp}$ del codex Vindobonensis (L) y su familia (BR) debe descartarse de acuerdo con las otras fuentes (Itin. Anton. Aug. 414.4 y CIL $1132811.11 ; 32821.11$ y 32831.11 ).

${ }^{19}$ Los Vasos de Vicarello señalan 23 mp para este trayecto (CIL XI 3281 1. 11; $32821.11 ; 32831.11$ ). 


$\begin{array}{cclcc}413 & 1 & \text { Item ab Hispali Cordubam mp XCIII sic } & 2021 \\ 2 & \text { Obucula } & \text { mp XLII } & 22 \\ 3 & \text { Astigi } & \text { mp XV } & 23 \\ 4 & \text { Ad Aras } & \text { mp XII } & 24 \\ 5 & \text { Corduba } & \text { mp XXIIII } & 25\end{array}$

Ptolomeo señala cuatro de las cinco poblaciones de esta vía: "I $\sigma \pi \alpha \lambda ı /$ His-

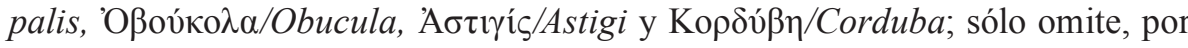

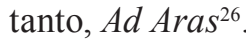

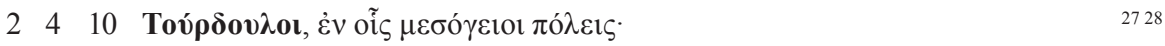

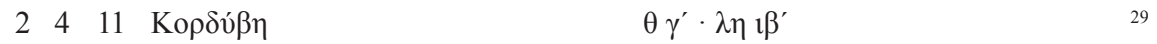

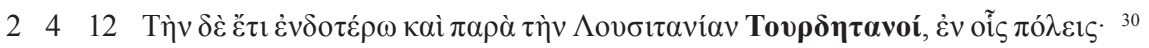

2414 'Т $\sigma \pi \alpha \lambda 1 \varsigma \quad \zeta \delta^{\prime} \cdot \lambda \zeta \mathrm{L} \gamma^{\prime}$

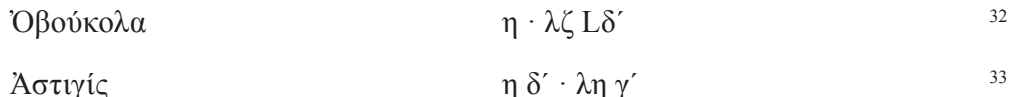

${ }^{20}$ Seguimos las ediciones de Wesseling (1735), Parthey - Pinder (1848) y Cuntz (1929). Codices: D: Parisinus Regius 7230 A (s. X). L: Vindobonensis (s. VIII), con correcciones de una segunda mano ( $\left.\mathbf{L}^{2}\right)$ y una tercera $\left(\mathbf{L}^{3}\right)$. B: Parisiensis Regius (s. IX). R: Florentinus (s. X). C: Parisiensis Regius (s. XII). (vid. Cuntz 1929: IV-VII).

${ }^{21}$ Item ab Hispali Cordubam] om. C. Hispali] Nespali D. Cordubam] Corduba LBR. XCIII] XCIIII $\mathbf{L}, \mathbf{X C I I I ~} \mathbf{L}^{3}$.

${ }^{22}$ Obucula mp XLII] om. C. Obucula] Abuccula BR. XLII] LXII D.

${ }^{23}$ Astigi mp XV] om. C. XV] XVI LBR Wesseling Parthey \& Pinder; la distancia de LBR debe ser corregida en XV de acuerdo con la otra cita en la vía X: XV mp (ITin. Anton. Aug. 414.4); y en los Vasos de Vicarello: XV mp (CIL XI 3281, 1. 9; 3282, 1. 9; y 3283, 1. 9).

${ }^{24}$ Ad Aras mp XII] om. C. XII] XV vel XII L, XVI Wesseling.

${ }^{25}$ Corduba mp XXIIII] om. C. XXIIII] XXII D.

${ }^{26}$ Robert C. Knapp (1993: 108) identifica esta mansio con "A $\sigma 0 \lambda \alpha$ (Ptol. Geog. 2.4.14).

${ }^{27}$ Trabajamos con las ediciones de Wilberg (1838), Nobbe (1843), Müller (1883) y Stückelberger Grasshoff (2006) y hemos consultado los códices V, R, X y A disponibles en la página web de la Universidad de Berna (http://www.philoscience.unibe.ch/projects/show-all/478-ptolemy-manuscripts).

Codices: న: U: Vaticanus Urbinas Graecus 82 (s. XIII). K: Seragliensis GI 57 (s. XIII). V: Vaticanus Graecus 177 (s. XIII). R: Marcianus Graecus 516 (s. s. XIV). || X: X: Vaticanus Graecus 191 (s. XIII). \| A: Vaticanus Palatinus Graecus 388 (s. XV). O: Florentinus Laurentianus XXVIII, 49 (s. XIV). (vid. Stückelberger \& Grasshoff 2006: 46).

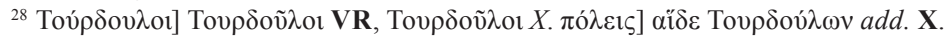

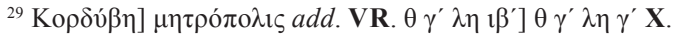

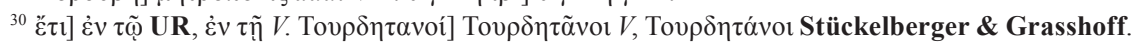

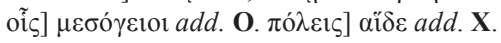

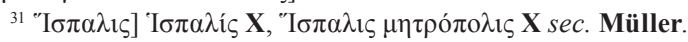

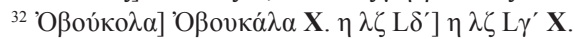


La comparación de las distancias documentadas por el Itinerario y las deducidas de la obra de Ptolomeo para cada uno de los trayectos de la vía sólo muestra una similitud, la distancia entre Hispalis y Obucula, $42 \mathrm{mp}$ en el Itinerario y 37,4 en la Geographia (vid. Tabla 1).

Tabla 1. Comparativa de las distancias (mp) de los trayectos en el Itinerario, Ptolomeo y la realidad.

\begin{tabular}{|c|c|c|c|c|c|c|c|c|c|}
\hline \multicolumn{2}{|c|}{ Itinerario } & \multicolumn{4}{|c|}{ Ptolomeo } & \multicolumn{4}{|c|}{ Real $^{34}$} \\
\hline Nombre & $m p^{35}$ & Nombre & $\lambda^{36}$ & $\varphi$ & $\mathrm{mp}$ & Nombre & $\lambda$ & $\varphi$ & $\mathrm{mp}$ \\
\hline Hispalis & 93 & "I $\sigma \pi \alpha \lambda 1 \varsigma$ & 7,25 & 37,83 & 103,8 & Sevilla & $-5,99$ & 37,39 & 80,9 \\
\hline Obucula & 42 & 'Оßои́ко $\lambda \alpha$ & 8,00 & 37,75 & 37,4 & Monclova & $-5,32$ & 37,52 & 41,2 \\
\hline Astigi & 15 & 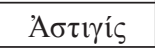 & 8,25 & 38,33 & 38,5 & Écija & $-5,08$ & 37,54 & 14,4 \\
\hline Ad Aras & 12 & & & & & La Carlota & $-4,95$ & 37,65 & 11,3 \\
\hline Corduba & 24 & 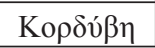 & 9,33 & 38,08 & 55,4 & Córdoba & $-4,78$ & 37,88 & 20,0 \\
\hline
\end{tabular}

Sin embargo, si comparamos los datos a partir de un punto de referencia fijo, el origen de la vía, las medidas deducibles de las dos fuentes se igualan. Así, las distancias partiendo de Hispalis son: a Obucula, $42 \mathrm{mp}$ según el Itinerario y 37,4 mp según Ptolomeo; a Astigi, 57 y 58,3 mp respectivamente; y, a Corduba, 93 y $103,8 \mathrm{mp}$ (vid. Tabla 2$)$.

Tabla 2. Distancias (mp) desde Hispalis y Córdoba al resto de poblaciones de la vía VIII según el Itinerario (It), Ptolomeo (Pt) y la realidad (Real)

\begin{tabular}{|c|c|c|c|c|c|c|}
\hline \multicolumn{3}{|c|}{ Desde Hispalis $\downarrow$} & \multicolumn{5}{c|}{ Via VIII } \\
\hline Real & $\mathrm{Pt}$ & It & Hispalis & 93 & 103,8 & 80,9 \\
\hline 41,2 & 37,4 & 42 & Obucula & 51 & 69,0 & 42,0 \\
\hline 55,5 & 58,3 & 57 & Astigi & 36 & 55,4 & 31,2 \\
\hline 65,1 & - & 69 & Ad Aras & 24 & - & 20,0 \\
\hline 80,9 & 103,8 & 93 & Corduba & It & Pt & Real \\
\cline { 6 - 8 } & & & & \multicolumn{2}{|c|}{ Desde Corduba } \\
\cline { 5 - 7 }
\end{tabular}

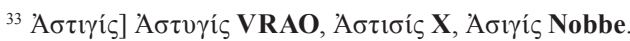

${ }^{34}$ Las localizaciones son las expuestas en el Barrington Atlas of the Greek and Roman World (Talbert 2000): Hispalis en Sevilla, Obucula en La Monclova (Fuentes de Andalucía), Astigi en Écija, $A d$ Aras en La Carlota y Corduba en Córdoba.

${ }^{35}$ Las equivalencias que utilizamos son $1 \mathrm{mp}=8 \mathrm{st}=1480 \mathrm{~m}$.

${ }^{36}$ Coordenadas en grados decimales de las poblaciones, $\lambda$ (longitud) y $\varphi$ (latitud), a partir de las cuales calculamos las distancias mediante la Ecuación 1. 
Estos datos muestran que Ptolomeo no partió de los trayectos parciales para situar las poblaciones de esta vía, como se había planteado (vid. Knapp 1993), sino de un punto de referencia fijo, Hispalis.

\section{ANÁLISIS DE LAS POSICIONES}

La combinación de las informaciones dadas por el propio Ptolomeo sobre sus fuentes, las deducciones sobre el probable método utilizado y la evidencia de las distancias nos permiten plantear una interpretación de las posiciones de los puntos señalados.

Ptolomeo ubicaría en primer lugar Hispalis: así lo muestra su condición de punto de referencia, si bien el método y los datos utilizados por el autor para ello son difíciles de precisar. Las coordenadas no permiten suponer un cálculo aritmético de su posición (vid. nota 9) y las distancias que la separan de los posibles puntos de referencia de la costa (vid. nota 8) no coinciden con las informaciones que tenemos (vid. Gómez Fraile 2005: 48-49) ${ }^{37}$. Por ello, o suponemos que hubo otra fuente que relacionara Hispalis con algún lugar más de la costa, posiblemente de la vertiente mediterránea ${ }^{38}$, o su posición dependió de algún otro núcleo del interior, quizá Emerita $^{39} \mathrm{o}$ tal vez una población del mismo recorrido como Corduba o Astigi ${ }^{40}$.

${ }^{37}$ Las fuentes sólo transmiten dos posibles puntos de referencia en la costa: la desembocadura del Baetis y el portus Gaditanus; ambos en la vertiente atlántica, y las informaciones sobre ellos no coinciden con las deducibles de la Geographia. Estrabón sitúa Hispalis a 500 st (62,5 mp) de la desembocadura del Baetis (Str. 3.2.3) y Ptolomeo a 108,5 mp, encontrándose, en realidad, próxima a la distancia señalada por el primero, a unos $90 \mathrm{~km}$ siguiendo el río (aprox. $60 \mathrm{mp}$ ). Respecto al puerto Gaditano, el Itinerario da una distancia de $76 \mathrm{mp}$ (ITin. Anton. Aug. 409-410) y de Ptolomeo se deducen 112,6 mp, si lo identifi-

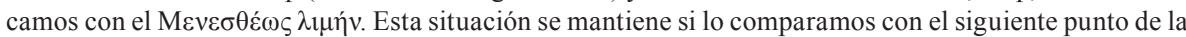
vía de identificación segura, Hasta/'A $\sigma \tau \alpha$, respecto a la que el Itinerario señala $60 \mathrm{mp}$ y de Ptolomeo se deducen $81 \mathrm{mp}$. A pesar de esta diferencia, Knapp (1993: 107-108) cree que Hispalis se ubicó a partir de Hasta y justifica las distintas mediciones por un error de Ptolomeo, que contabilizaría dos veces el trayecto entre Hasta y Ugia de $27 \mathrm{mp}$.

${ }^{38}$ La posición de Hispalis en la Geographia resulta demasiado oriental respecto a la costa atlántica, sin embargo, las distancias a los puntos del litoral mediterráneo se aproximan a los valores reales, así, por ejemplo, al Ká $\lambda \pi \eta$ ópos (Peñón de Gibraltar) se deducen 99,7 mp de las coordenadas ptolemaicas y en la realidad hay unos 149,5 km en línea recta (aprox. $101 \mathrm{mp}$ ).

${ }^{39}$ La distancia entre Hispalis y Emerita en la Geographia es de 110,4 mp y en el Itinerario de $123 \mathrm{mp}$ (Itin. Anton. Aug. 413 y 432), sin contabilizar el trayecto de Italica al Mons Mariorum de $46 \mathrm{mp}$ que parece ser erróneo, pues en línea recta hay unos $172 \mathrm{~km}$ (aprox. 116,2 mp). Descartamos otras posibilidades como la de Castulo por las diferencias de las medidas, según Ptolomeo entre Hispalis y Castulo hay 132,1 mp y según el Itinerario $171 \mathrm{mp}$ (ITIN. Anton. Aug. 403, 404 y 413), en línea recta hay unos $220,5 \mathrm{~km}$ (aprox. $149 \mathrm{mp})$

${ }^{40}$ Esto supondría un trabajo de localización simultáneo para varias poblaciones, algo que creemos verosímil teniendo presente que el autor partiría de un diseño previo que le permitiera esbozar el recorrido de las vías. 
Corduba sería la segunda población ubicada de la vía. Su posición pudo determinarse gracias a la localización de Hispalis, al sentido de la vía, deducible de la posición relativa de las respectivas etnias de las dos poblaciones, de los Turdetani al oeste de los Turduli, y a la distancia itineraria entre ambas, $93 \mathrm{mp}$. Ptolomeo pudo establecer la posición final del núcleo con esta información mediante la intersección de una circunferencia a escala de $93 \mathrm{mp}$ con centro en Hispalis y una recta hacia el Este redondeando el resultado al paralelo y meridano de 5 ' más próximo. Este redondeo podría explicar la diferencia entre las medidas deducibles de la obra de Ptolomeo y las documentadas por el Itinerario, 103,8 y $93 \mathrm{mp}$, si bien, creemos que es más probable que en ella interviniera alguna otra información itineraria que relacionara Corduba con algún otro núcleo destacado y que obligara al autor a modificar su posición intentando que se adecuara lo mejor posible a todos los datos que de ella poseía.

A continuación, Ptolomeo pudo situar las otras dos poblaciones de la vía, Obucula y Astigi, siguiendo el mismo sistema que para Corduba.

La posición de Obucula no presenta problemas de interpretación y su posición responde perfectamente a los planteamientos expuestos. Así, podemos suponer que Ptolomeo, una vez realizado el mismo trabajo gráfico que el descrito para Corduba, redondeó su posición al paralelo y meridiano de 5 ' más próximo, lo que finalmente la llevó a un paralelo ligeramente al sur del de su población de referencia.

La localización de Astigi, al igual que la de Corduba, pudo verse afectada por informaciones itinerarias que la relacionaban con otros núcleos ajenos a la vía estudiada, lo que, aparentemente, obligó a Ptolomeo a desplazarla hacia el Norte ${ }^{41}$ (vid. figura 1) distorsionando la distancia respecto a Corduba pero manteniendo con precisión la separación respecto a Hispalis, ya que se sitúa a 58,3 mp de ella y el Itinerario documenta $57 \mathrm{mp}$ (vid. Tabla 2).

Ptolomeo

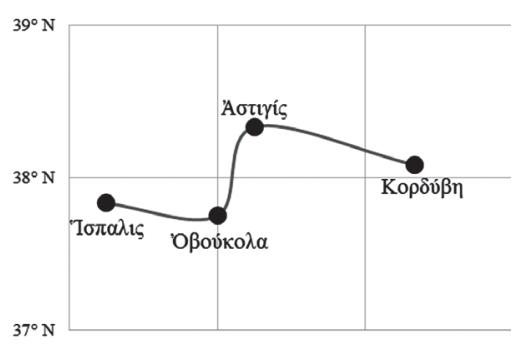

Realidad

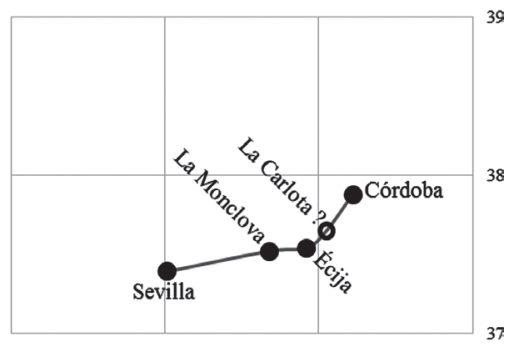

Figura 1. esquema de la via VIII sobre una retícula euclidiana del mapa de Ptolomeo y la realidad

${ }^{41}$ Esta posición septentrional también puede observarse en la distancia entre Emerita y Astigi, 73,9 mp en la Geographia y 105 mp según el Itinerario (IтıN. Anton. Aug. 414-415). 


\section{Conclusiones}

Cumpliendo con el objetivo del presente estudio, hemos deducido las fuentes y el posible método utilizado por Ptolomeo para situar las poblaciones de la conocida como vía VIII de Hispania. Así, constatamos que:

1. Las fuentes utilizadas por Ptolomeo para la localización de las poblaciones de la vía sólo fueron de tipo geométrico. El autor lograría ubicar los núcleos gracias a la combinación de los datos ofrecidos por, al menos, dos fuentes de este tipo: una descripción corográfica relativa a las etnias y un itinerario terrestre.

1.1. La descripción corográfica señalaría o permitiría deducir la posición relativa de los Turduli al este de los Turdetani.

1.2. El itinerario terrestre incluiría las distancias de los núcleos de la vía entre sí o, al menos, respecto a un punto de referencia, previsiblemente, Hispalis.

1.3. Es posible que Ptolomeo utilizara una fuente de tipo astronómico para situar el punto del que parte la localización de Hispalis, quizá Emerita, aunque no hay mayores evidencias.

2. El método seguido por Ptolomeo incluyó un trabajo previo y una serie de operaciones gráficas simples.

2.1. El trabajo previo consistió en el trazado de una retícula regional, la señalización en ella de los puntos con informaciones astronómicas absolutas y el diseño general de los límites provinciales y principales elementos geográficos.

3. Ptolomeo, gracias a ellos, sólo necesitó ubicar uno de los puntos de la vía, Hispalis, y la orientación general del recorrido hacia el Este para localizar sus núcleos.

3.1. La localización de Hispalis pudo partir de algún punto con informaciones astronómicas asociadas como Emerita, si bien los datos de los que disponemos no nos permiten confirmalo.

3.2. La orientación de la vía hacia el Este pudo deducirse de la posición relativa de los Turduli, etnia del destino de la vía, Corduba, al este de los Turdetani, etnia de su origen, Hispalis.

4. Las operaciones gráficas permitieron establecer la posición del resto de las poblaciones de la vía. Así, su localización se determinaría mediante la intersección de una circunferencia con centro en Hispalis de radio igual a la distancia entre las poblaciones con la recta que esbozara la orientación general del recorrido, redondeando el resultado al paralelo y meridiano de 5' más próximo. Este cálculo pudo estar condicionado por otros factores, límites o fronteras y el trazado de otras vías, lo que justifica el desplazamiento de $A s$ tigi hacia el Norte. 
APÉNDICE:

TABLAS DE DISTANCIAS ENTRE POBLACIONES

Tabla 3. Distancias (mp) entre las poblaciones en el Itinerario

\begin{tabular}{|c|c|c|c|c|c|}
\cline { 2 - 6 } \multicolumn{1}{c|}{} & Hispalis & Obucula & Astigi & Ad Aras & Corduba \\
\hline Hispalis & $\bullet$ & 42 & 57 & 69 & 93 \\
\hline Obucula & 42 & $\bullet$ & 15 & 27 & 51 \\
\hline Astigi & 57 & 15 & $\bullet$ & 12 & 36 \\
\hline Ad Aras & 69 & 27 & 12 & $\bullet$ & 24 \\
\hline Corduba & 93 & 51 & 36 & 24 & $\bullet$ \\
\hline
\end{tabular}

Tabla 4. Distancias (mp) entre las poblaciones en la Geographia

\begin{tabular}{|c|c|c|c|c|c|c|c|}
\hline & \multirow{2}{*}{$\begin{array}{c}\text { "І } \sigma \pi \alpha \\
\text { Codd }\end{array}$} & \multicolumn{2}{|c|}{ 'Оßои́ко $\lambda \alpha$} & \multirow{2}{*}{$\frac{A \sigma \tau \imath \gamma}{\text { Codd }}$} & \multicolumn{2}{|c|}{ 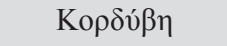 } \\
\hline & & & $\Omega$ & $X$ & & $\Omega$ & $\mathrm{X}$ \\
\hline & & $\begin{array}{c}7^{\circ} 15 \\
37^{\circ} 50^{\prime}\end{array}$ & $\begin{array}{c}8^{\circ} \\
37^{\circ} 45^{\prime}\end{array}$ & $\begin{array}{c}8^{\circ} \\
37^{\circ} 50^{\prime}\end{array}$ & $\begin{array}{c}8^{\circ} 15^{\prime} \\
38^{\circ} 20^{\prime}\end{array}$ & $\begin{array}{l}9^{\circ} 20^{\prime} \\
38^{\circ} 5^{\prime}\end{array}$ & $\begin{array}{c}9^{\circ} 20^{\prime} \\
38^{\circ} 20^{\prime}\end{array}$ \\
\hline "I $\sigma \pi \alpha \lambda ı \varsigma$ & Codd & $\bullet$ & 37,4 & 37,0 & 58,3 & 103,8 & 107,1 \\
\hline \multirow{2}{*}{ 'Оßои́ко $\lambda \alpha$} & $\Omega$ & 37,4 & $\bullet$ & 5,2 & 38,5 & 69,0 & 75,1 \\
\hline & $\mathrm{X}$ & 37,0 & 5,2 & $\bullet$ & 33,6 & 67,5 & 72,7 \\
\hline 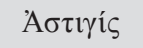 & Codd & 58,3 & 38,5 & 33,6 & $\bullet$ & 55,4 & 53,1 \\
\hline \multirow{2}{*}{ 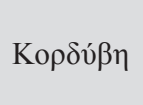 } & $\Omega$ & 103,8 & 69,0 & 67,5 & 55,4 & $\bullet$ & 15,6 \\
\hline & $X$ & 107,1 & 75,1 & 72,7 & 53,1 & 15,6 & $\bullet$ \\
\hline
\end{tabular}

Tabla 5. Distancias (mp) entre las poblaciones en la realidad

\begin{tabular}{|c|c|c|c|c|c|}
\hline & Sevilla & Monclova & Écija & La Carlota & Córdoba \\
\hline Sevilla & $\bullet$ & 41,2 & 55,5 & 65,1 & 80,9 \\
\hline Monclova & 41,2 & $\bullet$ & 14,4 & 24,1 & 42,0 \\
\hline Écija & 55,5 & 14,4 & $\bullet$ & 11,3 & 31,2 \\
\hline La Carlota & 65,1 & 24,1 & 11,3 & $\bullet$ & 20,0 \\
\hline Córdoba & 80,9 & 42,0 & 31,2 & 20,0 & $\bullet$ \\
\hline
\end{tabular}




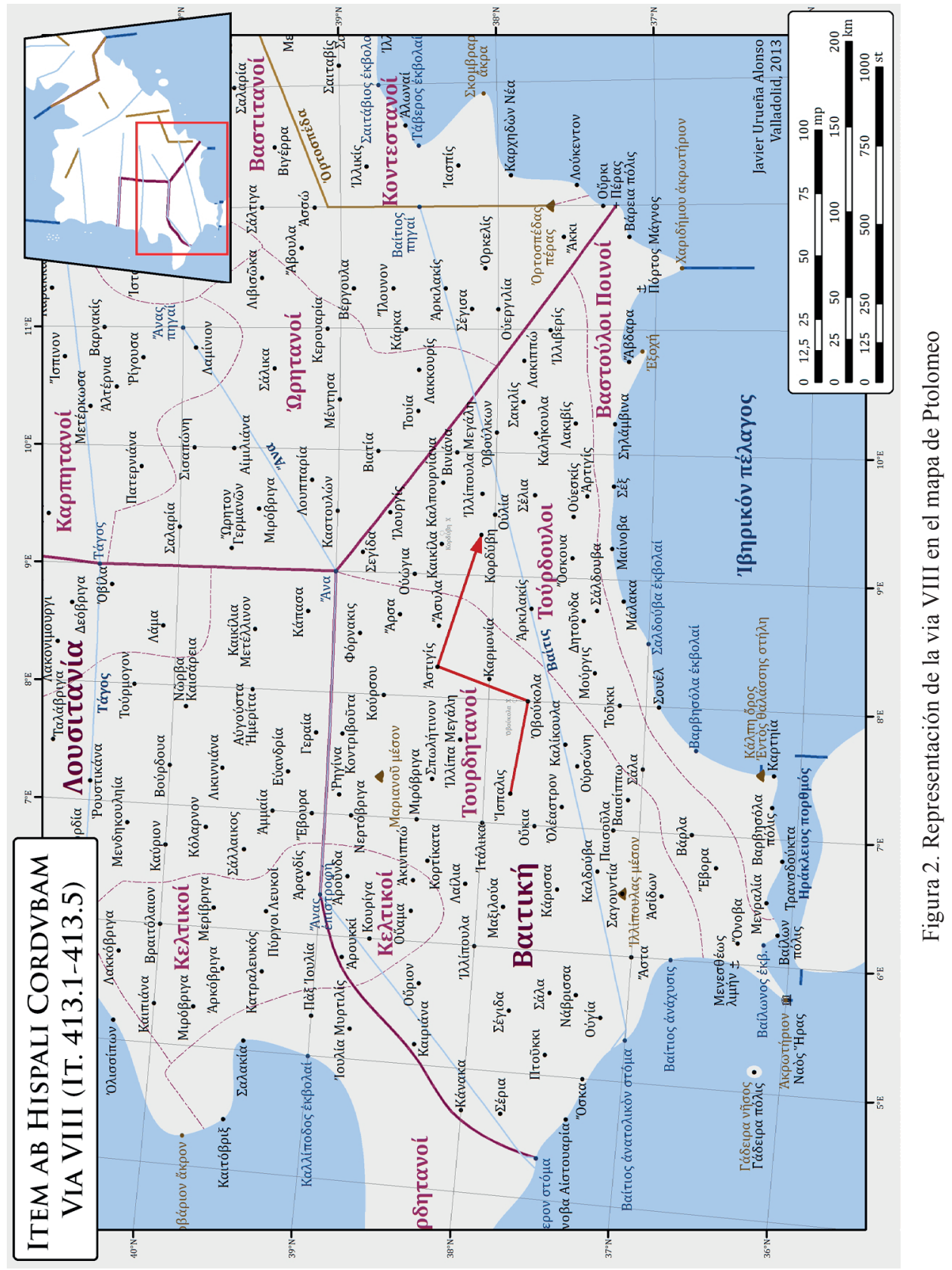




\section{BIBLIOGRAFÍA}

Blázquez 1892: A. Blázquez, "Nuevo estudio sobre el Itinerario de Antonino", BRAH 21 (1892) 54-128.

Bunbury 1879: E. H. Bunbury, A History of Ancient Geography (Vol. 2) (London 1879).

Canto 1997: A. M. Canto y de Gregorio, “Una nueva imagen de Ptolomeo: hipótesis de ubicación de ciudades vasconas", en Pueblos, lenguas y escrituras en la Hispania prerromana (Zaragoza 1997) 339-358.

Capalvo 1996: A. Capalvo Liesa, Celtiberia: un estudio de fuentes literarias antiguas (Zaragoza 1996).

Cuntz 1923: O. Cuntz, Die Geographie des Ptolemaeus: Galliae Germania Raetia Noricum Pannoniae Illyricum Italia (Berlin 1923).

Cuntz 1929: O. Cuntz (Ed.), Itineraria Romana (Leipzig 1929).

Gómez Fraile 1997: J. M. Gómez Fraile, 'La geografía de la Hispania Citerior en C. Tolomeo: análisis de sus elementos descriptivos y aproximación a su proceso de elaboración", Polis 9 (1997) 183-247.

Gómez Fraile 2005: J. M. Gómez Fraile, "Sobre la antigua cartografía y sus métodos: Los fundamentos numéricos de la Hispania de Claudio Ptolomeo", Iberia 8 (2005) 35-64.

Knapp 1993: R. C. Knapp, "Ptolomeo y la red viaria de la Bética”, en Actas del I Coloquio de Historia Antigua de Andalucia (Vol. 2) (Córdoba 1988) 103-110.

Martínez Hombre 1964: E. Martínez Hombre, Vindius. El lado septentrional clásico en Hispania (Madrid 1964).

Monteagudo 1947: L. Monteagudo, "Galicia en Ptolomeo", CuadGallegos 8 (1947) 609-653.

Montero Vítores 1991: J. Montero Vítores, Carpetanos y vettones en la Geografia de Ptolomeo (Madrid 1991).

Müller 1855: K. Müller (Ed.), Geographi Graeci minores (Vol. 1) (Paris 1855).

Müller 1883: K. Müller (Ed.), Claudii Ptolemaei Geographia (Vol. 1) (Paris 1883).

Nobbe 1843: K. F. A. Nobbe (Ed.), Claudii Ptolemaei Geographia (Vol. 1) (Leipzig 1843).

Parthey \& Pinder 1848: G. Parthey, M. Pinder (Eds.), Itinerarium Antonini Augusti et Hierosolymitanum (Berlin 1848).

Saavedra 2000: E. Saavedra, Descripción de la Via Romana entre Uxama y Augustobriga (Madrid 2000).

Sidoli 2004: N. Sidoli, Ptolemy's Mathematical Approach: Applied Mathematics in the Second Century (Toronto 2004).

Solana Sáinz 1972: J. M. Solana Sáinz, "Ensayos sobre el valor del grado Ptolemaico", HAnt 2 (1972) 69-76.

Stückelberger \& Grasshoff 2006: A. Stückelberger, G. Grasshoff (Eds.), Ptolemaios Handbuch der Geographie (Vols. 1-2) (Basilea 2006). 
Talbert 2000: R. J. A. Talbert (ed.), Barrington Atlas of the Greek and Roman World (Vol. 2) (Princeton 2000).

Tornadijo Rodríguez 2008a: T. F. Tornadijo Rodríguez, "Confirmación de la posición de "Flavionavia" por igualación de áreas de triángulos esféricos", Investigaciones geográficas 47 (2008) 175-184.

Tornadijo Rodríguez 2008b: T. F. Tornadijo Rodríguez, "Transformaciones de longitud y latitud para Ptolomeo. Addenda et corrigenda", El Nuevo Miliario 6 (2008) 24.

Tsorlini 2009: A. Tsorlini, "Higher order systematic effect in Ptolemy's Geographia coordinate description of Iberia", e-Perimetron 4.2 (2009) 117-130.

Wesseling 1735: P. Wesseling (Ed.), Vetera Romanorum Itineraria sive Antonini Augusti Itinerarium cum notis, Itinerarium Hierosolymitanum et Hieroclis Synecdemus (Amsterdam 1735).

Wilberg 1838: F. W. Wilberg (Ed.), Claudii Ptolomaei Geographiae libri octo: graece et latine ad codicum manu scriptorum fidem (Essen 1838). 